$62^{\text {ème }}$ Congrès de la SFCO, 03001 (2014)

DOI: $10.1051 /$ sfco/20146203001

(C) Owned by the authors, published by EDP Sciences, 2014

\title{
Prise en charge chirurgicale des manifestations bucco-dentaires chez une patiente atteinte d'un syndrome de Sturge-Weber.
}

\author{
Guillet $\mathbf{J}^{\mathbf{1}}$, Curien $\mathbf{R}^{\mathbf{1}}$, Mainard $\mathbf{L}^{\mathbf{2}}$, Bracard $\mathbf{S}^{3}$
}

1 Service d'Odontologie, CHU Nancy, Rue du Morvan, 54500, Vandoeuvre-les-Nancy, FRANCE

2 Service de Neurologie Pédiatrique, CHU Nancy, Hôpital d'Enfants, Site de Brabois, 54500, Vandoeuvre-les-Nancy, FRANCE

3 Service de Neuroradiologie, CHU Nancy, Hôptal central, 54000, Nancy, FRANCE

Le syndrome de Sturge-Weber est une malformation vasculaire congénitale non héréditaire, caractérisée par un angiome plan facial plus ou moins étendu, des anomalies lepto-méningées souvent responsables de comitialité dans l'enfance, de retards dans les acquisitions. L'angiome plan facial est généralement unilatéral et suit la distribution d'une ou plusieurs branches du nerf trijumeau (V). Les manifestations buccales sont variables mais fréquentes : hyperplasie gingivale liée à l'atteinte vasculaire, halitose, accidents d'éruption, etc... Par ailleurs, la prescription de certains traitements anti-épileptiques peut aggraver I'hyperplasie gingivale.

Le cas présenté ici est celui d'une jeune fille de 20 ans, atteinte d'un syndrome de Sturge-Weber complet avec retard mental. Elle présente un angiome plan de la face touchant les territoires du V1, du V2, du V3, et franchissant la ligne médiane. Une macro-chéilie mandibulaire importante accompagne le tableau clinique ; cela empêche la coaptation labiale et conduit à une ventilation buccale. Elle a également développé une atteinte endo-buccale caractérisée par une hypertrophie gingivale sévère du côté homolatéral à l'angiome, recouvrant les dents, et occasionnant des traumatismes répétés par morsure. II en résulte des saignements pluri-quotidiens et nocturnes, ne permettant plus le maintien d'une hygiène bucco-dentaire convenable. La jeune patiente est sous trithérapie anti-épileptique mais l'atteinte unilatérale de l'hypertrophie gingivale a fait éliminer une cause médicamenteuse.
La patiente a été prise en charge de façon pluridisciplinaire au $\mathrm{CHU}$ de Nancy ; une angio-IRM a été réalisée sous anesthésie générale afin d'objectiver la présence d'éléments vasculaires au sein des zones d'hypertrophie qu'il fallait supprimer. Une biopsie gingivale a été effectuée lors de cette même séance. Une décision collégiale d'intervention a ensuite été prise entre neurologues, neuro-radiologues, chirurgiens maxillo-faciaux infantiles et odontologistes. La patiente a été opérée dans le service d'Odontologie du CHU de Nancy sous anesthésie générale, afin de réaménager le parodonte et de réaliser les avulsions dentaires nécessaires.

Le syndrome de Sturge-Weber est une pathologie rare mais dont les conséquences bucco-dentaires sont fréquentes. Une hygiène buccale déficiente aggrave la situation en raison de l'inflammation qu'elle engendre. II est donc capital pour ces patients de bénéficier d'un suivi régulier afin de limiter la survenue de complications, notamment infectieuses.
GUILLET Julie j.guillet@chu-nancy.fr

This is an Open Access article distributed under the terms of the Creative Commons Attribution License 4.0, which permits unrestricted use, distribution, and reproduction in any medium, provided the original work is properly cited. 